\title{
Assessment of the ratio of pollen to seed flow in a cline for genetic variation in a quantitative trait
}

\author{
XIN-SHENG HU ${ }^{1 *} \&$ BAILIAN LI ${ }^{2}$ \\ ${ }^{1}$ Department of Forestry, University of British Columbia, 3rd Floor, 3041-2424 Main Hall, \\ Vancouver, BC, V6T 1Z4, Canada \\ ${ }^{2}$ Department of Forestry, College of Forest Resources, North Carolina State University, PO Box 8002, \\ Raleigh, NC 27695-8002, U.S.A.
}

\begin{abstract}
A dispersal-selection cline model is analysed to evaluate the role of the ratio of pollen to seed flow $(r)$ in spatial genetic variation, with a focus on clines in additive and dominant variances of major genes affecting a quantitative trait, assuming one locus with two alleles, no genetic drift and no mutation. It is shown that under weak selection, steady-state departures from the value at Hardy-Weinberg equilibrium (HWE) for genotype frequency $(D)$ and additive variance $\left(\Delta V_{\mathrm{a}}\right)$ generally display a small value on one side of the selection boundary point, a high value around the point, and a moderate value on the other side of the boundary point. A large value of $r$ can enhance formation of this pattern. However, this is not the case for clines in the average effect of a gene substitution $(\Delta \alpha)$ or in dominance variance $\left(\Delta V_{\mathrm{d}}\right)$, where large values of $r$ can eradicate the clines. Direct use of their values at HWE to approximate the real case is acceptable. There is an expected turning point that divides clines of either additive variance or dominance variance into two subclines, each with a shorter width than that of allele frequency. Integration of these properties can help to indicate the existence of major genes affecting a quantitative trait.
\end{abstract}

Keywords: additive variance, cline, dominance variance, pollen flow, seed flow.

\section{Introduction}

An important type of genetic structure observed in natural populations is clinal variation, where gene frequency or morphological traits exhibit a gradient change (increase or decrease) with geographical distance. Clines are usually formed in hybridization zones induced by primary intergradation of populations within a continuous distribution, or by secondary contact of isolated populations at one time (Endler, 1977; Mayr, 1979). Distinction between these two types of origination is still a challenge to us today, especially when a cline at steady state is reached. According to the involvement of dispersal or not, clines can be generally grouped into two types, dispersal-independent and dispersal-dependent clines. Both types have been observed in many natural populations of animal and plant species (Endler, 1977; Barton \& Hewitt, 1985). Theoretical studies on the type of dispersal-dependent cline go back to Fisher's pioneering work (Fisher, 1937), who

*Correspondence. E-mail: xhu@interchange.ubc.ca analysed the wave of advance of advantageous genes. Since then, theoretical studies in this area have been extensive, and in most of these studies, the diffusion model is employed to mimic cline formation.

Early practical studies of natural clines concentrated on changes in morphological and physiological traits. For example, a cline in additive genetic variation of viability was observed in Drosophila melanogaster, and was successfully explained using dispersal-independent mechanisms (Kusakabe \& Mukai, 1984; Mukai, 1988). With molecular technology making DNA markers available for precisely locating quantitative trait loci (QTLs), genetic bases for the evolutionary forces acting on QTLs will be understood in the future. By employing appropriate DNA markers and relevant statistical models, many studies have uncovered the existence of major genes that contribute to a large proportion of genetic variation of quantitative traits, e.g. the stem growth and form in Populus (Bradshaw \& Stettler, 1995), the volume growth and wood specific gravity in Eucalyptus grandis (Grattapaglia et al., 1995), and the wood specific gravity in Pinus taeda (Groover et al., 1994). Thus, use of these QTL markers or major genes 
to address the clinal situation can help to elucidate the process whereby clines exist for quantitative traits.

Recently, cline theory has been extended to plant populations subdivided for diploid nuclear genes. Nagylaki (1997) showed that by altering model parameters, existing cline theory becomes suitable for describing the behaviour of diploid plant nuclear genes. However, it only focused on the cline in allele frequency, and hence cannot reflect characteristics of clines in genetic variance, although genetic variance is a function of allele frequency. It is also shown that migration can maintain more variation within populations and less between populations for a quantitative trait with additive effects only (Wright, 1969; Cockerham \& Tachida, 1987). However, the clinal variation in genetic variance for additive-dominant traits with major genes has not been considered in a dispersal-dependent cline, although reports on clines of different quantitative traits are extensive (Barton \& Gale, 1993).

The ratio of pollen to seed flow is used for understanding the relative contributions to migration between seed and pollen flows. The biological bearings of the ratio were stressed in elucidating the discrepancy in population genetic structure for genes with different modes of inheritance (Ennos, 1994; Hu \& Ennos, 1997, 1999). There are several practical examples showing how to estimate the ratio (Ennos, 1994). In the same way, estimation of the ratio in clines can also be carried out because clinal variation is only a specific type of population genetic structure. However, the role of the ratio is not examined in shaping clines in genetic variation, even though it was applied to explain cline concordance and discordance between phenotypic trait and maternally inherited markers (Young, 1996). Thus, it is interesting to evaluate the ratio in clines for a quantitative trait via exploring the general behaviour of its major genes, provided that major genes exist for the trait. The aim of this study is to assess the ratio of pollen to seed flow in shaping clines in terms of different genetic statistics.

\section{The model}

\section{Assumptions}

A single locus with two alleles $\left(A_{1}, A_{2}\right)$ is considered for a diploid nuclear gene. A hermaphrodite plant species is distributed in an infinite chain of equally spaced populations each with population size large enough so that the effects of genetic drift can be ignored. Migration is symmetrical between populations, such that the migration between populations $i$ and $j(i \neq j)$ is $m_{i j}=m_{j i}(i$ or $j=0, \pm 1, \pm 2, \ldots)$. The life cycle for each population follows a sequence of events: pollen flow, random mating, seed flow, and selection. These events are assumed to occur within a short time interval $\Delta t$. The population distribution is assumed to be uniform after selection, and hence $m_{i j}$ represents the probability of migration from population $j$ to $i$ in the time $\Delta t$. Random combination between pollen and ovules is assumed in each population. Density-independent selection takes place at each location independently after seed flow.

It is necessary to note here in advance that the genes, addressed in clines for allele substituting effect, additive and dominance variances in this paper, refer to major genes affecting a quantitative trait. The results obtained in other cases can also be applied to other type of genes that do not contribute to the variation of a quantitative trait.

\section{Allele frequency}

For the sake of simplicity, the partial differential equation for allele frequency is briefly described. Let $p(i, t)$ be the gene frequency of allele $A_{1}$ in population $i$ at time $t$, and $q(i, t)$ for allele $A_{2}(p(i, t)+q(i, t)=1)$. Suppose that the number of pollen grains produced by adults within the time interval $\Delta t$ is large enough that the frequency in pollen, $p_{\mathrm{P}}(i, t+\Delta t)$, is the same as in adults, i.e. $p_{\mathrm{P}}(i, t+\Delta t)=p(i, t)$. After pollen flow, the gene frequency in pollen in population $i$ is $p_{\mathrm{P}}^{*}(i, t+\Delta t)=\sum_{j} m_{\mathrm{P} \cdot i j} p_{\mathrm{P}}(j, t+\Delta t)$, where $m_{\mathrm{P} \cdot i j}$ is the migration rate of pollen from population $j$ to $i$, and $\sum_{j} m_{\mathrm{P} \cdot i j}=1$.

After random combination between pollen and ovules and formation of seeds, it can be shown that the gene frequency in seeds, $p_{\mathrm{S}}(i, t+\Delta t)$, can be expressed by $p_{\mathrm{S}}(i, t+\Delta t)=\left[\begin{array}{ll}1 & \sum_{j \neq i} m_{\mathrm{P} \cdot i j} / 2\end{array}\right] p(i, t)+\left(\sum_{j \neq i} m_{\mathrm{P} \cdot i j} / 2\right)$. $p(j, t)$. By ignoring all second order forms for migration rate, $O\left(m_{\mathrm{P}}^{2}\right)$, the frequencies of three genotypes after seed flow can be simply expressed by $\left(1+\sum_{j \neq i} m_{\mathrm{S} \cdot i j}\right)$ $p_{\mathrm{S}}^{2} \quad(i, t+\Delta t)+\sum_{j \neq i} m_{\mathrm{S} \cdot i j} p_{\mathrm{S}}^{2}(j, t+\Delta t)$ for $A_{1} A_{1}, \quad 2(1$ $\left.\sum_{j \neq i} m_{\mathrm{S} \cdot i j}\right) p_{\mathrm{S}}(i, t+\Delta t) q_{\mathrm{S}}(i, t+\Delta t)+2 \sum_{j \neq i} m_{\mathrm{S} \cdot i j} \quad p_{\mathrm{S}}$ $(j, t+\Delta t) q_{\mathrm{S}}(j, t+\Delta t)$ for $A_{1} A_{2}$, and $\left(1 \quad \sum_{j \neq i} m_{\mathrm{S} \cdot i j}\right)$ $q_{\mathrm{S}}^{2}(i, t+\Delta t)+\sum_{j \neq i} m_{\mathrm{S} \cdot i j} q_{\mathrm{S}}^{2}(j, t+\Delta t)$ for $A_{2} A_{2}$, where $m_{\mathrm{S} \cdot i j}$ is the migration rate of seed from population $j$ to $i$, and $\sum_{j} m_{\mathrm{S} \cdot i j}=1$. For simplicity, denote these frequencies, respectively, by $f_{11}^{\prime}(t+\Delta t), 2 f_{12}^{\prime}(t+\Delta t)$ and $f_{22}^{\prime}(t+\Delta t)$ for population $i$. Thus, by ignoring all items containing the second order of migration rate, $O\left(m_{\mathrm{S}}^{2}, m_{\mathrm{P}}^{2}\right)$, and the product term for migration rates of seed and pollen, $O\left(m_{\mathrm{S}} m_{\mathrm{P}}\right)$, we can obtain

$$
\begin{aligned}
f_{11}^{\prime}(t+\Delta t)= & \left(1 \sum_{j \neq i}\left(m_{\mathrm{S} \cdot i j}+m_{\mathrm{P} \cdot i j}\right)\right) p^{2}(i, t) \\
& +\sum_{j \neq i} m_{\mathrm{P} \cdot i j} p(j, t)(p(i, t)+p(j, t))
\end{aligned}
$$




$$
\begin{array}{rl}
f_{12}^{\prime}(t+\Delta t)= & \left(1 \sum_{j \neq i}\left(m_{\mathrm{S} \cdot i j}+m_{\mathrm{P} \cdot i j}\right) / 2\right) p(i, t) \\
& +\sum_{j \neq i}\left(m_{\mathrm{S} \cdot i j}+m_{\mathrm{P} \cdot i j} / 2\right) p(j, t) \\
& f_{11}^{\prime}(t+\Delta t) \\
f_{22}^{\prime}(t+\Delta t)=1 & 2\left(1 \sum_{j \neq i}\left(m_{\mathrm{S} \cdot i j}+m_{\mathrm{P} \cdot i j}\right) / 2\right) p(i, t) \\
& 2 \sum_{j \neq i}\left(m_{\mathrm{S} \cdot i j}+m_{\mathrm{P} \cdot i j} / 2\right) p(j, t) \\
+ & f_{11}^{\prime}(t+\Delta t)
\end{array}
$$

Using Wrightian fitnesses $1+s \Delta \operatorname{tg}(i), 1+h s \Delta \operatorname{tg}(i)$, and $1-s \Delta \operatorname{tg}(i)$ for genotypes $A_{1} A_{1}, \quad A_{1} A_{2}$, and $A_{2} A_{2}$, respectively, where $h$ is degree of dominance, the mean fitness of population $i$ can be calculated $\bar{w}(i, t+\Delta t)=1+s \Delta t g(i)\left(f_{11}^{\prime}(t+\Delta t)+2 h f_{12}^{\prime}(t+\Delta t)\right.$ $\left.f_{22}^{\prime}(t+\Delta t)\right)$. Ignoring all items for the second order of migration and equivalents, $O\left(s^{2}, s m_{\mathrm{S}}, s m_{\mathrm{P}}, m_{\mathrm{P}}^{2}, m_{\mathrm{S}}^{2}\right.$, $\left.m_{\mathrm{S}} m_{\mathrm{P}}\right)$, the allele frequency after selection can be calculated by

$$
\begin{aligned}
p(i, t+\Delta t)=(1 & \left.\sum_{j \neq i}\left(m_{\mathrm{S} \cdot i j}+m_{\mathrm{P} \cdot i j} / 2\right)\right) p(i, t) \\
& +\sum_{j \neq i}\left(m_{\mathrm{S} \cdot i j}+m_{\mathrm{P} \cdot i j} / 2\right) p(j, t)+s \Delta \operatorname{tgp}(i, t) \\
& \times\left(\begin{array}{lll}
1 \quad p(i, t))(1+h & 2 h p(i, t))
\end{array}\right.
\end{aligned}
$$

According to the assumptions mentioned above, $m_{\mathrm{S} \cdot i j}+m_{\mathrm{P} \cdot i j} / 2$ is equivalent to the transition probability from state (population here) $j$ to $i$ in a Markov process (Feller, 1971; p. 322). The assumption of the uniform distribution indicates that a diffusion model can be used to approximate homogeneous migration. Scale location of population by $x=i \varepsilon$, where $\varepsilon$ is the spacing between populations. Conditions similar to (8) of Nagylaki (1978) are postulated. By applying (9) of Nagylaki (1978), we can readily obtain

$\frac{\partial p}{\partial t}=\frac{\sigma^{2}}{2} \frac{\partial^{2} p}{\partial x^{2}}+\operatorname{sg}(x) p(1 \quad p)(1+h \quad 2 h p)$

where $\sigma^{2}=\sigma_{\mathrm{S}}^{2}+\sigma_{\mathrm{P}}^{2} / 2, \sigma_{\mathrm{S}}^{2}$ and $\sigma_{\mathrm{P}}^{2}$ are dispersal variances of seed and pollen, respectively, and $p$ refers to $p(x, t)$. Equation 3 is the same as that in a specific case (without selfing) obtained by Nagylaki (1997), who used a more sophisticated method to address plant populations.

\section{Steady disequilibrium coefficient}

Denote frequencies of three genotypes after selection by $f_{11}(t+\Delta t), 2 f_{12}(t+\Delta t)$, and $f_{22}(t+\Delta t)$ for population $i$. It can be shown that

$$
\begin{aligned}
& f_{11}(t+\Delta t)=f_{11}^{\prime}(t+\Delta t)+2 s \Delta t g(i) p^{2}(i, t) \\
& \times(1 \quad p(i, t))(1 \quad h p(i, t))+O\left(s^{2}\right) \\
& f_{12}(t+\Delta t)=f_{12}^{\prime}(t+\Delta t)+s \Delta t g(i) p(i, t) \\
& \times(1 \quad p(i, t))(1+h)(1 \quad p(i, t))^{2} \\
& \text { (1 h) } \left.p^{2}(i, t)\right)+O\left(s^{2}\right) \\
& f_{22}(t+\Delta t)=f_{22}^{\prime}(t+\Delta t) \\
& 2 s \Delta t g(i) p(i, t)(1 \quad p(i, t))^{2} \\
& \times(1+h(1 \quad p(i, t)))+O\left(s^{2}\right)
\end{aligned}
$$

By substituting eqn 1a into eqn $4 \mathrm{a}$ and using the diffusion approximation, we can obtain

$$
\begin{aligned}
f_{11}(t+\Delta t)= & p^{2}+2 s \Delta t g(x) p^{2}\left(\begin{array}{lll}
1 & p
\end{array}\right)\left(\begin{array}{ll}
1 & h p
\end{array}\right) \\
& +\frac{\sigma_{\mathrm{S}}^{2}+\sigma_{\mathrm{P}}^{2}}{2} \frac{\partial^{2}}{\partial x^{2}} p^{2} \Delta t .
\end{aligned}
$$

Denote the departure from Hardy-Weinberg equilibrium (HWE) at generation $t$ by $D \Delta t$, scaled by the time interval $\Delta t$. We can obtain $f_{11}(t)=p^{2}+D \Delta t$. Applying it into eqn 5 and defining $\partial f_{11} / \partial t=0$, a steady-state value for the disequilibrium coefficient is

$D=2 s g(x) p^{2}(1 \quad p)(1 \quad h p)+\left(\sigma_{\mathrm{S}}^{2}+\sigma_{\mathrm{P}}^{2}\right) / 2 \cdot \partial^{2} p^{2} / \partial x^{2}$.

Suppose that the function $g(x)$ in an infinite cline is equal to 1 , when $x<0$, and $-\beta^{2}$ when $x>0$, when $\beta^{2}$ is the ratio of selection intensities in the two parts of habitat. Using the relationship $\partial^{2} p^{2} / \partial x^{2}=2(\partial p / \partial x)^{2}+$ $2 p\left(\partial^{2} p / \partial x^{2}\right)$, the steady disequilibrium coefficient for either side of the boundary point is calculated by

$$
\begin{aligned}
D(0+)= & 2 s \beta^{2} p^{2}\left(\begin{array}{ll}
q(1 & h p
\end{array}\right) \\
& \left.4 \cdot \frac{1+r}{2+r}\left(1+h \quad 5(1+3 h) p / 6+3 h p^{2} / 2\right)\right) \\
& \text { for } x>0 ;(6 \mathrm{a})
\end{aligned}
$$

$$
\begin{gathered}
D(0 \quad)=2 s p^{2} q(1 \quad h p)+4 \frac{1+r}{2+r} \\
s\left(\begin{array}{lll}
\frac{1}{3} & 2 p^{2}(1+h & \left.5(1+3 h) p / 6+3 h p^{2} / 2\right)
\end{array}\right) \\
\quad \text { for } x<0
\end{gathered}
$$

(c) The Genetics Society of Great Britain, Heredity, 87, 400-409. 
where $r=\sigma_{\mathrm{P}}^{2} / \sigma_{\mathrm{S}}^{2}$, the ratio of dispersal variance of pollen to seed. $D$ is a linear function of $s$ for a given set of $p, h$ and $r$. The weak selection can lead to a minor departure from HWE. It can be seen that at the selection boundary point, which can be calculated using the method introduced by Haldane (1948), $\lim _{x \rightarrow 0+} D(0+)$ is not equal to $\lim _{x \rightarrow 0+} D(0)$. Thus, the point at $x=0$ is an interrupt point in the distribution of $D$ with geographical distance. At the boundary point, a relationship of $D(0-)=-D(0+) / \beta^{2}+4(1+r) s / 3(2+r)$ can be derived. If $r=0, D(0-)=-D(0+) / \beta^{2}+2 s / 3$. If $r \rightarrow \infty, D(0-)=-D(0+) / \beta^{2}+4 s / 3$. A large value of $r$ can lead to a great divergence in $D$-value between two sides of the boundary point.

The preceding analysis can be depicted more clearly for a given gene frequency interval [0.1, 0.9] (Fig. 1). Parameter settings are used to mimic the case where the heterozygote is selected. Although the disequilibrium coefficient is small owing to the weak selection, all populations located at $x>0$ exhibit heterozygote deficiency $(D(0+)>0$, while some populations at $x<0$ possess an excess of heterozygotes $(D(0-)<0$. This is because allele frequencies in these populations satisfy a condition of $\left.\left.r>1 \quad 3 p^{2} q / 2\left(\begin{array}{lll}1 & (6 & 5 p\end{array}\right) p^{2}\right)\right)^{1} 2$ according to eqn $6 \mathrm{~b}$.

\section{Steady cline for the average effect of allele substitution}

The method used by Falconer (1989) is employed to derive the average effect of an allele substitution. After seed flow and selection, HWE is violated in each population. For a given allele $A_{1}$, the probability for the occurrence of $A_{1} A_{1}$ and $A_{1} A_{2}$ is $P\left(A_{1} A_{1} \mid A_{1}\right)$ and $P\left(A_{1} A_{2} \mid A_{1}\right)$, respectively. Similarly, the probability for the occurrence of $A_{1} A_{1}$ and $A_{1} A_{2}$ for a given allele $A_{2}$ is $P\left(A_{1} A_{1} \mid A_{2}\right)$ and $P\left(A_{1} A_{2} \mid A_{2}\right)$, respectively. According to Bayesian formulae and using diffusion approximation, we can show that

$P\left(A_{1} A_{1} \mid A_{1}\right)=p+c_{1}, \quad P\left(A_{1} A_{2} \mid A_{1}\right)=1 \quad p \quad c_{1}$

$P\left(A_{1} A_{2} \mid A_{2}\right)=p+c_{2}, \quad P\left(A_{2} A_{2} \mid A_{2}\right)=1 \quad p \quad c_{2}$

where $c_{1}=s \Delta \operatorname{tg}(x) \Delta t p(1-p)(1-h)+\left(\sigma_{\mathrm{P}}^{2} / 4\right) \partial^{2} p / \partial x^{2}$, and $c_{2}=s \Delta t g(x) \Delta t p(1-p)(1+h)+\left(\sigma_{\mathrm{P}}^{2} / 4\right) \partial^{2} p / \partial x^{2}$.

Denote the genetic values of three genotypes by $a$ for $A_{1} A_{1}, d$ for $A_{1} A_{2}$, and $-a$ for $A_{2} A_{2}$. Population mean is thus calculated by $M=(p-q) a+2(p q-D \Delta t) d=$ $M_{\mathrm{E}}-2 D d \Delta t$, where $M_{\mathrm{E}}$ is the value at HWE. Breeding value of allele $A_{1}, \alpha_{1}$, is equal to $P\left(A_{1} A_{1} \mid A_{1}\right) a+$ $P\left(A_{1} A_{2} \mid A_{1}\right) d-M$, and $\alpha_{2}$ for allele $A_{2}$ is $-P\left(A_{2} A_{2} \mid A_{2}\right) a+P\left(A_{1} A_{2} \mid A_{2}\right) d-M$. Thus, the average allele substitution effect, $\alpha$, is $\alpha_{1}-\alpha_{2}$. Similarly, let $\alpha(t)=\alpha_{\mathrm{E}}+\Delta \alpha \Delta t$, where $\alpha_{\mathrm{E}}=a+(q-p) d$, the value at HWE. $\Delta \alpha$ is the increment within time interval $\Delta t$. The steady-state departure of $\alpha$ from the HWE value in the time interval $\Delta t(\partial \alpha / \partial t=0)$ is derived as,

$$
\begin{aligned}
& \Delta \alpha(0+)=2 s \beta^{2} p q\left(h a+\left(\begin{array}{lll}
1 \quad \frac{r}{2+r}(1+h \quad 2 h p)
\end{array}\right) d\right) \\
& \text { when } x>0 ;(8)
\end{aligned}
$$

Replacing $\beta^{2}$ in eqn 8 with -1 , it then becomes the result when $x<0$. It is also true that $\lim _{x \rightarrow 0+} \Delta \alpha(0+) \neq$ $\lim _{x \rightarrow 0} \Delta \alpha(0)$, an interrupt point at $x=0$.

Numerical examples are analysed in the case with complete dominance for a major gene $(a=d=1.0)$. Although the expected allele substituting effect $\alpha_{E}$ at HWE gradually increases with geographical distance, behaviours of $\Delta \alpha(0+)$ and $\Delta \alpha(0-)$ are different due to
Fig. 1 Impacts of the ratio of pollen to seed flow $(r)$ on the change of the steadystate Hardy-Weinberg disequilibrium coefficient $(D)$ with geographical distance. Parameter settings are $s=0.01, h=0$, and $\sigma_{\mathrm{S}}^{2}=1.0$.

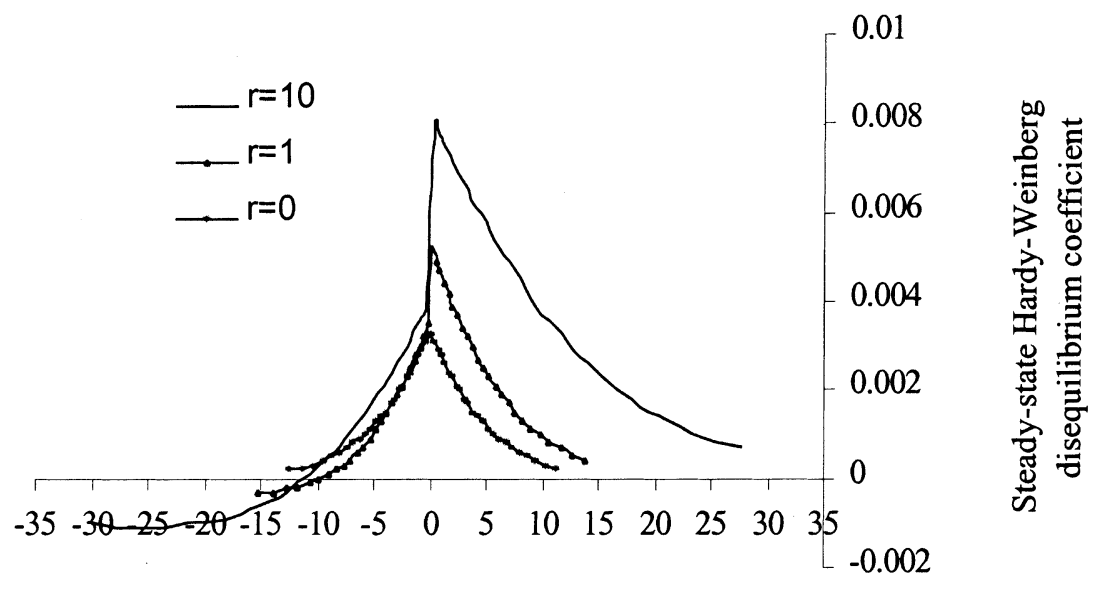

Geographical distance

(C) The Genetics Society of Great Britain, Heredity, 87, 400-409. 


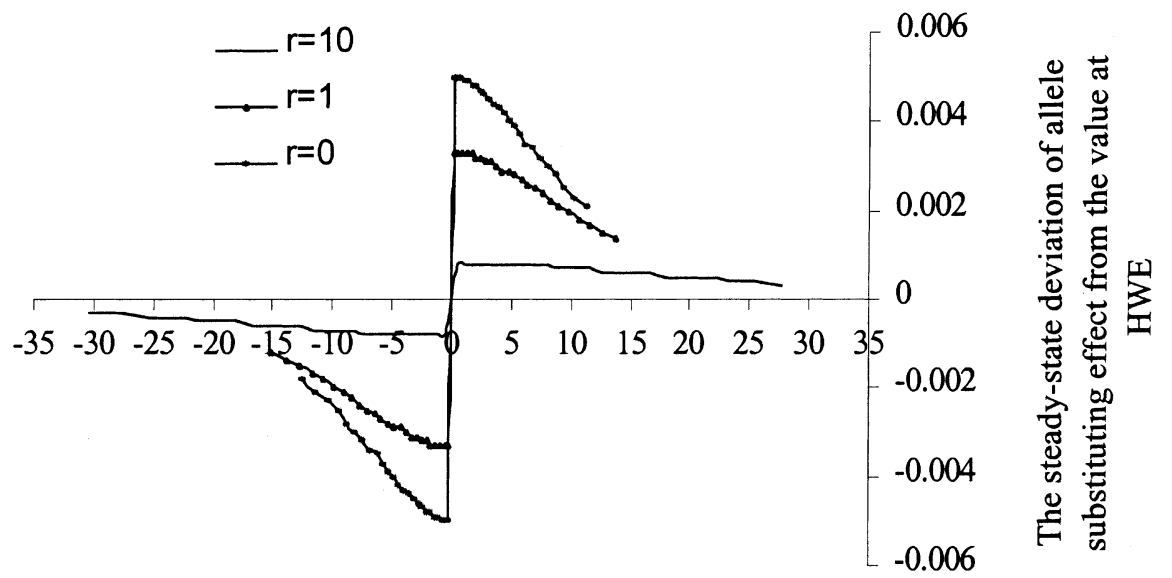

Geographical distance

the contrasting selections, with a positive value for $\Delta \alpha(0+)$ and a negative value for $\Delta \alpha(0-)$. However, the departure from $\alpha_{\mathrm{E}}$ is reduced with the ratio $r$ in all populations (Fig. 2).

\section{Steady additive variance}

According to the method used by Falconer (1989), the additive effect variance, $V_{\mathrm{a}}$, is $4 f_{11}(t+\Delta t) \alpha_{1}^{2}(t+\Delta t)$ $+2 f_{12}(t+\Delta t)\left(\alpha_{1}(t+\Delta t)+\alpha_{2}(t+\Delta t)\right)^{2}+4 f_{22}(t+\Delta t) \alpha_{2}^{2}$ $(t+\Delta t)$ for population $i$. Thus, it can be shown that the steady-state change of $V_{\text {a }}$ from the value at HWE $\left(V_{\mathrm{aE}}=2 p q \alpha_{\mathrm{E}}^{2}\right)$ in the time interval $\Delta t, \Delta V_{\mathrm{a}}$, can be obtained by letting $\partial V_{\mathrm{a}} / \partial t=0$, i.e.

$\Delta V_{\mathrm{a}}(0+)=A a^{2}+B d^{2}+C a d$

where $A=2\left(D(0+)+4 s \beta^{2} h p^{2} q^{2}\right), B=2\left(\begin{array}{ll}q & p\end{array}\right)\left(\begin{array}{ll}q & p\end{array}\right)$. $\left.D(0+)+4 s \beta^{2} p^{2} q^{2}(1 \quad(r / 2+r)(1+h \quad 2 h p))\right), C=4$ $\left(\begin{array}{ll}q & p\end{array}\right) D(0+)+8 s \beta^{2} p^{2} q^{2} 1+(q \quad p) h \quad(r / 2+r) \cdot \quad(1+$ $h \quad 2 h p)$ ), for $x>0$; and replacing $\beta^{2}$ in the expressions of $A, B$, and $\mathrm{C}$ with -1 , and $D(0+)$ with $D(0-)$ in eqn 9 , it becomes the result under the case of $x<0\left(\Delta V_{\mathrm{a}}(0-)\right)$.

It can be viewed from numerical examples that the clinal change in allele frequency can make possible the clinal change in additive variance $\left(V_{\mathrm{aE}}+\Delta V_{\mathrm{a}}\right)$ within a relatively small geographical region across the boundary point (Fig. 3a). The larger the ratio $r$ is, the wider the cline of additive variance becomes. However, there is a maximum point that divides the cline in additive variance into two subclines. The location for the maximum point depends upon the relative values of additive and dominant effects. The contrasting selection intensities can be reflected from the performance of $\Delta V_{\mathrm{a}}$ on both sides of the selection boundary point, with the occurrence of large departures from $V_{\mathrm{aE}}$ in populations located at $x>0$ and of small departures at $x<0$.
Fig. 2 Impacts of the ratio of pollen to seed flow $(r)$ on the change of the steady-state deviation of allele substituting effect from the value at Hardy-Weinberg equilibrium $(\Delta \alpha)$ with geographical distance. Parameter settings are $s=0.01, h=0, a=d=1.0$ and $\sigma_{\mathrm{S}}^{2}=1.0$.

A large value of $r$ can bring about a great value of $\Delta V_{\mathrm{a}}$. Another striking characteristic is that $\Delta V_{\mathrm{a}}$ increases with geographical distance approaching to the boundary point $x=0$ (Fig. 3b).

\section{Steady dominance variance}

The dominance derivation for each genotype is $d_{11}=$ $a-M-2 \alpha_{1}$ for $A_{1} A_{1}, d_{12}=d-M-\alpha_{1}-\alpha_{2}$ for $A_{1} A_{2}$, and $d_{22}=-a-M-2 \alpha_{2}$ for $A_{2} A_{2}$. By expanding $f_{11}(t+\Delta t) d_{11}^{2}+2 f_{12}(t+\Delta t) d_{12}^{2}+f_{22}(t+\Delta t) d_{22}^{2}$, we can show that the steady change for the departure of dominance variance from the value at HWE $\left(V_{\mathrm{dE}}=4 p^{2} q^{2} d^{2}\right)$ in the time interval $\Delta t, \Delta V_{\mathrm{d}}$, is

$\Delta V_{d}(0+)=4\left(\begin{array}{ll}p & q\end{array}\right)^{2} D(0+) d^{2}, \quad$ when $x>0$.

Replacing $\beta^{2}$ with -1 and $D(0+)$ with $D(0-)$ eqn 10 becomes the result for the case of $x<0$.

Unlike the cline in additive variance, the cline in dominance variance $\left(V_{\mathrm{dE}}+\Delta V_{\mathrm{d}}\right)$ exhibits a symmetric shape with a maximum value at $p=0.5$. The whole cline is composed of two subclines connected at the maximum point, each subcline possessing a property of monotonically decreasing with the distance away from the maximum point (Fig. 4a). However, the behaviour of $\Delta V_{\mathrm{d}}$ is very different on either side of the boundary point because it is a quadratic function of allele frequency, with different maxima at each side. A large value of $r$ can reduce $\Delta V_{\mathrm{d}}$ in populations located at $x<0$ but increase $\Delta V_{\mathrm{d}}$ in populations at $x>0$ (Fig. $4 \mathrm{~b}$ ).

\section{Discussion}

Several properties can be obtained from evaluating the ratio of pollen to seed flow in shaping clines in terms of the average effect of allele substitution, Hardy- 


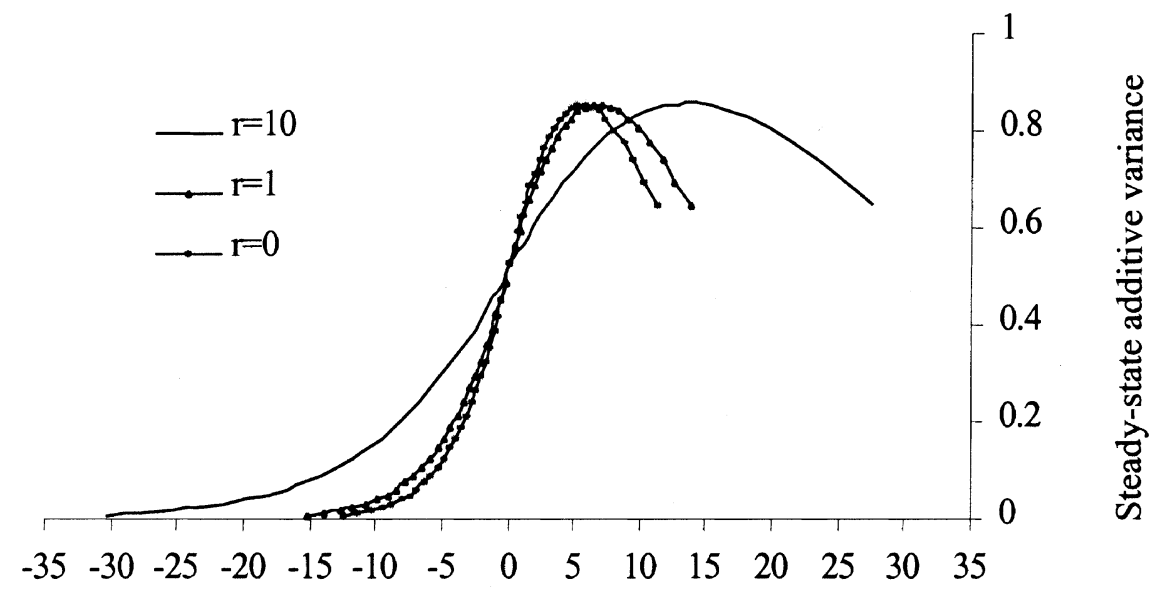

Fig. 3 Impacts of the ratio of pollen to seed flow ( $r$ ) on: (a) the change of the steady-state additive variance

( $V_{\mathrm{aE}}+\Delta V_{\mathrm{a}}$ ); and (b) the change of the steady-state deviation of additive variance from the value at HardyWeinberg equilibrium $\left(\Delta V_{\mathrm{a}}\right)$ with geographical distance. Parameter settings are $s=0.01, h=0, a=d=1.0$, and $\sigma_{\mathrm{S}}^{2}=1.0$.

Geographical distance

Weinberg disequilibrium coefficients, and variances of additive and dominance effects. One of these properties is that a continuous distribution of allele frequency cannot guarantee the continuity of clines in other genetic statistics investigated here at the boundary point. Traditionally, clines in allele frequencies and morphological traits were often studied, but clines in terms of genetic variances were not developed (Fisher, 1937; Haldane, 1948; Barton \& Hewitt, 1985; Barton \& Gale, 1993). Clines for additive genetic variance were recently developed in theory for quantitative traits without major genes (Barton, 1999). The existence of an interrupt point for clines in some genetic statistics at the boundary point has not been indicated in theory.

Gene flow can generate linkage disequilibria among loci in the dispersal-selection cline (Slatkin, 1975; Barton, 1983). Strong linkage disequilibria imply strong selection and high gene flow. Linkage disequilibria were hence proposed for the purpose of estimating the strength of selection in a cline (e.g. Barton \& Hewitt, 1985; Mallet et al., 1990). Similarly, Hardy-Weinberg disequilibria can also be generated for a single locus in the dispersal-selection cline (Barton \& Gale, 1993). Our theoretical results show that the Hardy-Weinberg disequilibrium coefficient is a linear function of the selection coefficient, but a nonlinear function of the ratio of pollen to seed flow. The slope for the change of Hardy-Weinberg disequilibrium coefficient with selection coefficient, $\partial D / \partial s$, is related to allele frequency and the ratio of pollen to seed flow (eqns $6 \mathrm{a}$ and $6 \mathrm{~b}$ ). A large ratio of pollen to seed flow can increase the slope $(\partial D / \partial s)$. Thus, the Hardy-Weinberg disequilibrium coefficient could be used for the purpose of estimating the selection coefficient as well, under certain conditions; especially for those populations that are located around the boundary point. However, Hardy-Weinberg disequilibrium coefficient could not be detected owing to the low statistical power in practice. This situation could occur especially in populations that are located far away 


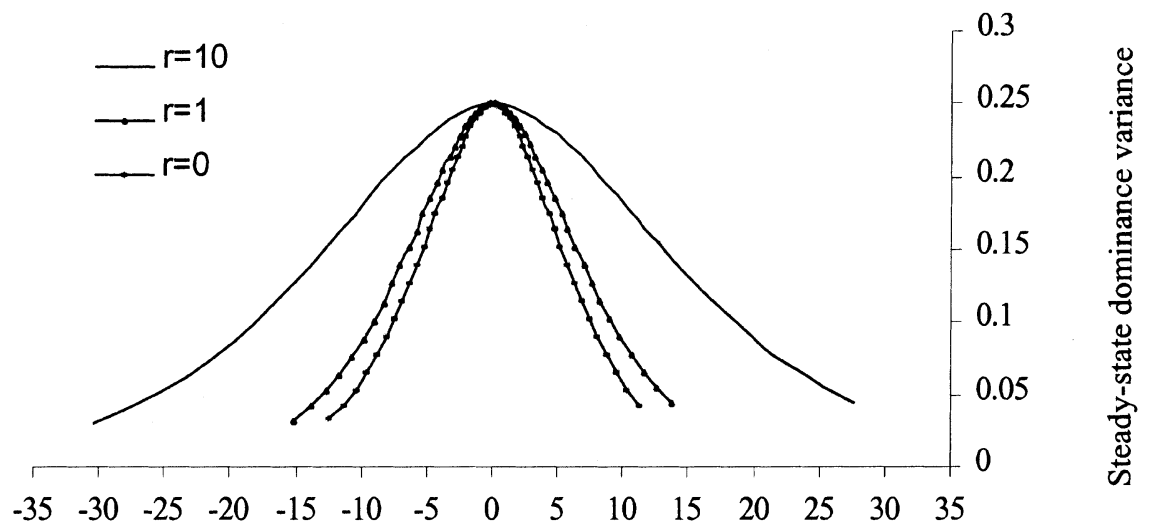

Geographical distance

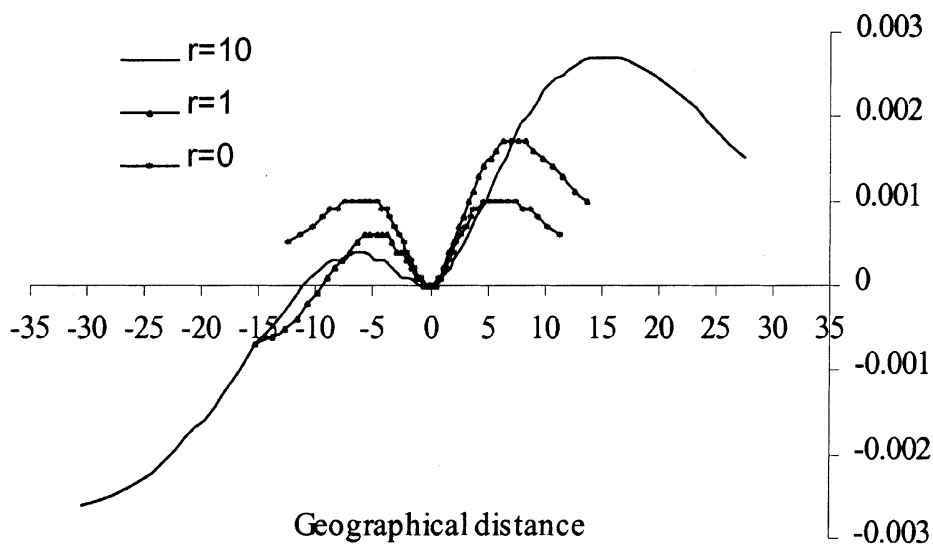

Fig. 4 Impacts of the ratio of pollen to seed flow $(r)$ on: (a) the change of the steady-state dominance variance $\left(V_{\mathrm{dE}}+\Delta V_{\mathrm{d}}\right)$; and (b) the change of the steady-state deviation of dominance variance from the value at Hardy-Weinberg equilibrium $\left(\Delta V_{\mathrm{d}}\right)$ with geographical distance. Parameter settings are $s=0.01, h=0, a=d=1.0$, and $\sigma_{\mathrm{S}}^{2}=1.0$. from the boundary point (e.g. Terauchi, 1990; Villani et al., 1999).

The properties for the cline in Hardy-Weinberg disequilibrium coefficient could improve our understanding of the characteristics underlying the dispersal-selection cline with one-step contrasting selections in habit. Hardy-Weinberg disequilibrium coefficient is changed from a small value in populations far away from one side of the boundary point, to a relatively high value in populations around the boundary point, and then to a moderate level in populations far away from another side of the boundary point (Fig. 1). The greatest divergence in Hardy-Weinberg disequilibrium coefficient is expected to occur between neighbouring populations separated by the boundary point. The divergence could be high for predominantly outcrossing species with large ratios of pollen to seed flow. Therefore, this property provides an alternative way to identify the boundary point in practical cline analysis.

Evidence supporting the above theoretical expectations comes from one typical dispersal-selection cline recently reported in the tree species Castanea sativa, a native widespread forest species in Europe (Villani et al.,
1999). Pollination in this species is carried out via insects and wind, and incompatibility systems prevent selffertilization. Some findings in natural populations of the species in Turkey are consistent with our theoretical expectation (Villani et al., 1999). Selection intensity acting on the cline was estimated to be 0.06 , six times as large as that used in our numerical examples. The level of heterozygote deficiency was 0.034 in western, 0.080 in Bithynian (around the boundary point), and 0.073 in eastern populations, generally displaying the spatial pattern similar to our theoretical expectations (Fig. 1). The greatest level of mean heterozygote deficiency among all 34 populations (0.07 in population Sapanca-Dibektas) was located just after boundary point $(x>0)$.

The theoretical results on the cline, in the average effect of allele substitution, also have implications for the analysis of the impact of allele substitution along a cline. The departure from the value at HWE for the average effect of allele substitution is a linear function of the selection coefficient. However, in practice, detection of this departure suffers from weak selection. Furthermore, a large ratio of pollen to seed flow will make the 
departure value approach zero. Therefore, direct use of the value at HWE for each population is reasonable in some real cases.

It would not be meaningful to consider the average effect of allele substitution for genes with small effects. However, in some cases major gene effects are due to multiple polymorphisms of a few individual sites that are located within the major gene, such as the level of alcohol dehydrogenase $(A d h)$ expression in Drosophila melanogaster (Stam \& Laurie, 1996). Combination of these individual effects can produce 'superalleles' with large effect. Under this situation, consideration on the average effect of 'superallele' substitution would be more useful than that of the individual 'allele' for the sites located within the major gene.

Our theoretical results also indicate that a spatial pattern similar to the clinal changes in Hardy-Weinberg disequilibrium coefficient is expected for the deviation of additive variance from the value at HWE. A large ratio of pollen to seed flow can enhance the formation of this spatial pattern. The deviation of additive variance from the value at HWE could probably be observed in practice since it is seriously affected by additive and dominant effects. Large additive and dominant effects in real cases can make the deviation of additive variance from the value at HWE detectable.

The model could provide a framework for quantitative assessment of the cline in additive variance of a single major gene. The cline in the additive variance is very different from that in allele frequency. The existence of a turning point (maximum point) in the cline of additive variance is probably an important feature. Location of the turning point depends on the combined effects of allele frequency, additive and dominant magnitudes, and the degree of dominance. This property is not possessed for the genetic variance cline in additive polygenic traits where a major gene does not exist (Barton, 1999). Moreover, different selection systems are assumed between the present study for a major gene and a previous study (Barton, 1999) for polygenes, each with infinitesimal effects.

There is only one empirical example that has addressed the clinal change for additive variance (Kusakabe \& Mukai, 1984; Mukai, 1988). However, no turning point was pointed out in the cline for the additive variance of viability in $D$. melanogaster, and only monotonic changes occurred from northern to southern populations. The cline was maintained by mutation-selection variance in northern populations and by diversifying selection in southern populations. A dispersal-selection system was not involved in its formation.

There are extensive studies of morphological clines across hybrid zones or clines where the dispersal- selection system is responsible for their formation (see review by Barton \& Hewitt, 1985). However, most analyses reduce trait variation to a discriminant function and concentrate on changes in means (e.g. Long \& Singh, 1995). Little empirical work has emphasized the quantitative genetics of clines (see also the discussion by Barton, 1999). The model presented here for the cline of additive variance provides additional theory for analysing the quantitative genetics of clines in the future.

Our theoretical results also imply some properties for the cline in dominance variance that has not been documented so far in the literature. Cline shape for dominance variance is different from that for additive variance, displaying a symmetric change with geographical distance. However, like the cline in additive variance, there is also a turning point in the cline in dominance variance, by which two contrasting subclines are divided. Departure for dominance variance from the value at HWE could not be detected when weak selection was involved in cline formation. Under this case, the cline for dominance variance at HWE can be used to approximate the real case. Again, there is little empirical work that has addressed the cline for dominance variance, but the present theory provides another aspect of properties for the cline in which a major gene affecting a quantitative trait is involved.

The above analyses imply that integration of these properties can gain a deep insight into the dispersalselection cline in which a major gene affecting a quantitative trait is involved. For most predominantly outcrossing species, such as many conifer species (Mitton, 1992), gene flow is mainly mediated by pollen grains, and the ratio of pollen to seed flow is often greater than 1.0 (Ennos, 1994; Ennos et al., 1999). The properties presented here could be strengthened for clines in terms of Hardy-Weinberg disequilibrium coefficient, average effect of allele substitution, and variances of additive and dominant effects.

Practically, it is difficult to evaluate the ratio of pollen to seed flow from phenotypic data at the population level, but the ratio can be estimated indirectly using selectively neutral markers with different modes of inheritance (Ennos, 1994; McCauley et al., 1996). Variances of additive and dominance effects can also be estimated according to conventional quantitative genetic theory (Falconer, 1989). Furthermore, if molecular markers that are tightly linked to QTLs with large effects are available, the linkage disequilibria among them could be maintained in the dispersal-selection cline (Li \& Nei, 1973; Slatkin, 1975). Use of such QTL markers for clinal analysis is expected in the future.

For polygenes each with equally infinitesimal effect, the aggregation behaviour over many genes is often concentrated and described in terms of mean and 
variance (Falconer, 1989). In practice, clines for individual genes with small effects are often undetectable. Thus, these comprehensive properties presented here cannot be applied for the aggregation behaviour of polygenes each with infinitesimal effect in a dispersaldependent cline. Some properties for clines in additive polygenic traits without major genes have been presented by Barton (1999), and are different from the properties presented here for a major gene. Therefore, existence of a major gene involving the formation of a cline could be indicated by integrating all information on the ratio of pollen to seed flow and the properties of clines in different genetic statistics investigated in the present model.

\section{Acknowledgements}

We greatly appreciate Dr Trudy Mackay at North Carolina State University and Dr Richard Ennos at the University of Edinburgh, for very helpful comments in preparation of this paper. Many thanks should be given to Dr John Brookfield, the designated editor, and two anonymous referees for comments that substantially improved the paper. This work is supported by a grant from the Department of Energy and the North Carolina State University-Industry Cooperative Tree Improvement Program.

\section{References}

BARTON, N. H. 1983. Multilocus clines. Evolution, 37, 454-471. BARton, N. H. 1999. Clines in polygenic traits. Genet. Res., 74, 223-236.

BARTON, N. H. AND GALE, K. S. 1993. Genetic analysis of hybrid zones. In: Harrison, R. G. (ed.) Hybrid Zones and the Evolutionary Process, pp. 13-45. Oxford University Press, New York.

BARTON, N. H. AND HEWITT, G. M. 1985. Analysis of hybrid zones. Ann. Rev. Ecol. Syst., 16, 113-146.

BRADSHAW, H. D. AND STETTLER, R. F. 1995. Molecular genetics of growth and development in Populus. IV. Mapping QTLs with large effects on growth, from and phenology in a forest tree. Genetics, 139, 963-973.

COCKERHAM, C. C. AND TACHIDA, H. 1987. Evolution and maintenance of quantitative genetic variation by mutations. Proc. Natl. Acad. Sci. U.S.A., 84, 6205-6209.

ENDLER, J. A. 1977. Geographic Variation, Speciation, and Clines. Princeton University Press, Princeton NJ.

ENNOS, R. A. 1994. Estimating the relative rates of pollen and seed migration among plant populations. Heredity, 72, 250-259.

ENNOS, R. A., SINCLAIR, W. T., HU, X. S. AND LANGDON, A. 1999. Using organelle markers to elucidate the history, ecology and evolution of plant populations. In: Hollingsworth, P. M., Bateman, R. M. and Gornall, R. J. (eds) Molecular
Systematics and Plant Evolution, pp. 1-19. Taylor \& Francis, London.

FALCONER, D. S. 1989. Introduction to Quantitative Genetics. Longman, Harlow, UK.

FELLER, w. 1971. An Introduction to Probability Theory and its Applications. Wiley, New York.

FISHER, R. A. 1937. The wave of advance of advantageous genes. Ann. Eugen., 7, 355-369.

GRATtAPAGLia, D., BERTOLUCCI, F. L. G. AND SEDEROFF, R. R. 1995. Genetic mapping of QTLs controlling vegetative propagation in Eucalyptus grandis and E. urophylla using a pseudo-testcross mapping strategy and RAPD markers. Genetics, 144, 1205-1214.

Groover, A., DEVy, M., FIDDLER, T., LEE, J. ET AL. 1994. Identification of quantitative trait loci influencing wood specific gravity in an outbred pedigree of loblolly pine. Genetics, 138, 1293-1300.

HALDANE, J. B. S. 1948. The theory of a cline. J. Genet., 28 , $277-284$.

HU, X. S. AND ENNOS, R. A. 1997. On estimation of the ratio of pollen to seed flow among plant populations. Heredity, 79, 541-552.

HU, X. S. AND ENNOS, R. A. 1999. Impacts of seed and pollen flow on population differentiation for plant genomes with three contrasting modes of inheritance. Genetics, 152, 441-450.

KUSAKABE, S. AND MUKAI, T. 1984. The genetic structure of natural populations of Drosophila melanogaster: Clinal and uniform genetic variation over populations. Genetics, 108, $617-632$.

LI, W. H. AND NEI, M. 1973. Stable linkage disequilibrium without epistasis in subdivided populations. Theor. Pop. Biol., 6, 173-183.

LONG, A. D. AND SINGH, R. S. 1995. Molecules versus morphology: the detection of selection acting on morphological characters along a cline in Drosophila melanogaster. Heredity, 74, 567-581.

MALleT, J., BARTON, N. H., LAMAS, M. G., SANTISTEBAN, C. J. ET AL. 1990. Estimates of selection and gene flow measures of cline width and linkage disequilibrium in Heliconius hybrid zones. Genetics, 124, 921-936.

MAYr, E. 1979. Animal Species and Evolution. Harvard University Press, Cambridge, MA.

MCCAUley, D. E., STEVENS, J. E., PERONI, P. A. AND RAVEILl, J. A. 1996. The spatial distribution of chloroplast DNA and allozyme polymorphisms within a population of Silene alba (Caryophyllaceae). Am. J. Bot., 83, 727-731.

MitTON, J. B. 1992. The dynamic mating systems of conifers. New Forests, 6, 197-216.

MUKAI, T. 1988. Genotype-environment interaction in relation to the maintenance of genetic variability in populations of Drosophila melanogaster. In: Weir, B. S., Goodman, M. M., Eisen, E. J. and Namkoong, G. (eds) Proceedings of the Second International Conference on Quantitative Genetics, pp. 21-31. Sinauer Associates, Sunderland, MA.

NAGYlaki, T. 1978. Random genetic drift in a cline. Proc. Natl. Acad. Sci. U.S.A., 75, 423-426.

NAGYLAKI, T. 1997. The diffusion model for migration and selection in a plant population. J. Math. Biol., 35, 409-431. 
SLATKIN, M. 1975. Gene flow and selection in a two-locus system. Genetics, 81, 787-802.

STAM, L. F. AND LAURIE, C. C. 1996. Molecular dissection of a major gene effect on a quantitative trait: the level of alcohol dehydrogenase expression in Drosophila melanogaster. Genetics, 144, 1559-1564.

TERAUCHI, R. 1990. Genetic diversity and population structure of Dioscorea tokoro Makino, a dioecious climber. Pl. Sp. Biol., 5, 243-254.

Villani, F., SANSOTTA, A., CHERUBini, M., CESARONI, D. ET AL. 1999. Genetic structure of natural populations of Castanea sativa in Turkey: evidence of a hybrid zone. J. Evol. Biol., 12, 233-244.

Wright, S. 1969. Evolution and the Genetics of Populations, vol. 2, The Theory of Gene Frequencies. Chicago, University of Chicago Press.

YounG, N. D. 1996. Concordance and discordance: a tale of two hybrid zones in the Pacific Coast irises (Iridaceae). Am. J. Bot., 83, 1623-1629. 